University of Nebraska - Lincoln

DigitalCommons@University of Nebraska - Lincoln

Public Health Resources

Public Health Resources

2005

\title{
Role of Elevated S-adenosylhomocysteine in Rat Hepatocyte Apoptosis: Protection by Betaine
}

\author{
Kusum K. Kharbanda \\ University of Nebraska Medical Center, kkharbanda@unmc.edu \\ David D. Rogers II \\ VA Alcohol Research Center, Department of Veterans Affairs Medical Center, Research Service (151), \\ 4101 Woolworth Avenue, Omaha, NE \\ Mark E. Mailliard \\ VA Alcohol Research Center, Department of Veterans Affairs Medical Center, Research Service (151), \\ 4101 Woolworth Avenue, Omaha, NE
}

Gerri L. Siford

Department of Internal Medicine, University of Nebraska Medical Center, Omaha, NE

Anthony J. Barak

VA Alcohol Research Center, Department of Veterans Affairs Medical Center, Research Service (151),

4101 Woolworth Avenue, Omaha, NE

See next page for additional authors

Follow this and additional works at: https://digitalcommons.unl.edu/publichealthresources

Part of the Public Health Commons

Kharbanda, Kusum K.; Rogers II, David D.; Mailliard, Mark E.; Siford, Gerri L.; Barak, Anthony J.;

Beckenhauer, Harriet C.; Sorrell, Michael F.; and Tuma, Dean J., "Role of Elevated S-adenosylhomocysteine in Rat Hepatocyte Apoptosis: Protection by Betaine" (2005). Public Health Resources. 36.

https://digitalcommons.unl.edu/publichealthresources/36

This Article is brought to you for free and open access by the Public Health Resources at DigitalCommons@University of Nebraska - Lincoln. It has been accepted for inclusion in Public Health Resources by an authorized administrator of DigitalCommons@University of Nebraska - Lincoln. 


\section{Authors}

Kusum K. Kharbanda, David D. Rogers II, Mark E. Mailliard, Gerri L. Siford, Anthony J. Barak, Harriet C.

Beckenhauer, Michael F. Sorrell, and Dean J. Tuma 


\title{
Role of elevated S-adenosylhomocysteine in rat hepatocyte apoptosis: Protection by betaine
}

\author{
Kusum K. Kharbanda ${ }^{\mathrm{a}, \mathrm{b}, *}$, David D. Rogers II ${ }^{\mathrm{a}}$, Mark E. Mailliard ${ }^{\mathrm{a}, \mathrm{b}}$, \\ Gerri L. Siford ${ }^{\mathrm{b}}$, Anthony J. Barak ${ }^{\mathrm{a}, \mathrm{b}}$, Harriet C. Beckenhauer ${ }^{\mathrm{a}}$, \\ Michael F. Sorrell ${ }^{\mathrm{a}, \mathrm{b}}$, Dean J. Tuma ${ }^{\mathrm{a}, \mathrm{b}}$ \\ ${ }^{a}$ VA Alcohol Research Center, Department of Veterans Affairs Medical Center, Research Service (151), \\ 4101 Woolworth Avenue, Omaha, NE 68105, USA \\ ${ }^{\mathrm{b}}$ Department of Internal Medicine, University of Nebraska Medical Center, Omaha, NE 68198, USA
}

Received 11 August 2005; accepted 22 September 2005

\begin{abstract}
Previous studies from our laboratory have shown that ethanol consumption results in an increase in hepatocellular S-adenosylhomocysteine levels. Because S-adenosylhomocysteine is a potent inhibitor of methylation reactions, we propose that increased intracellular S-adenosylhomocysteine levels could be a major contributor to ethanol-induced pathologies. To test this hypothesis, hepatocytes isolated from rat livers were grown on collagen-coated plates in Williams' medium E containing 5\% FCS and exposed to varying concentrations of adenosine in order to increase intracellular S-adenosylhomocysteine levels. We observed increases in caspase-3 activity following exposure to adenosine. This increase in caspase activity correlated with increases in intracellular S-adenosylhomocysteine levels and DNA hypoploidy. The adenosine-induced changes could be significantly attenuated by betaine administration. The mechanism of betaine action appeared to be via the methylation reaction catalyzed by betaine-homocysteine-methyltransferase. To conclude, our results indicate that the elevation of S-adenosylhomocysteine levels in the liver by ethanol is a major factor in altering methylation reactions and in increasing apoptosis in the liver. We conclude that ethanol-induced alteration in methionine metabolic pathways may play a crucial role in the pathologies associated with alcoholic liver injury and that betaine administration may have beneficial therapeutic effects.

Published by Elsevier Inc.
\end{abstract}

Keywords: Hepatocytes; S-adenosylhomocysteine; Adenosine; Betaine; Methylation; Apoptosis

\section{Introduction}

It has been demonstrated that ethanol administration is linked to increased hepatocyte apoptosis in both clinical and experimental alcohol-induced liver injury in a variety of species of animals including rats, mice and minipigs [113]. Although much progress has been made in understanding the role of critical factors that may be involved, such as cytokines and oxidative stress, there is still no unified hypothesis to explain the pathogenesis of alcoholinduced apoptosis of hepatocytes.

Our laboratory and others have established that multiple steps in the methionine metabolic cycle (Fig. 1) are

\footnotetext{
* Corresponding author. Tel.: +1 402346 8800x3752; fax: +1 4024490604 .

E-mail address: kkharbanda@unmc.edu (K.K. Kharbanda).
}

affected in the liver by ethanol consumption [14-16] The earliest studies conducted in our laboratory showed that a major defect elicited by ethanol consumption appears to be the inhibition of methionine synthase (MS) activity, resulting in impaired remethylation of homocysteine to form methionine $[3,17,18]$. This defect ultimately results in an increased generation of the potentially toxic agent, homocysteine, which is released from the liver [12-14,19-21]. Ethanol consumption also results in increased hepatocellular levels of S-adenosylhomocysteine (SAH) $[3,21,22]$ - a product of methyl transfer reactions involving S-adenosylmethionine (SAM) and the metabolic precursor of homocysteine and adenosine. Since the reaction that converts SAH to homocysteine and adenosine (catalyzed by SAH hydrolase) is reversible and proceeds toward hydrolysis only if the products are removed [15], the accumulation of homocysteine follow- 


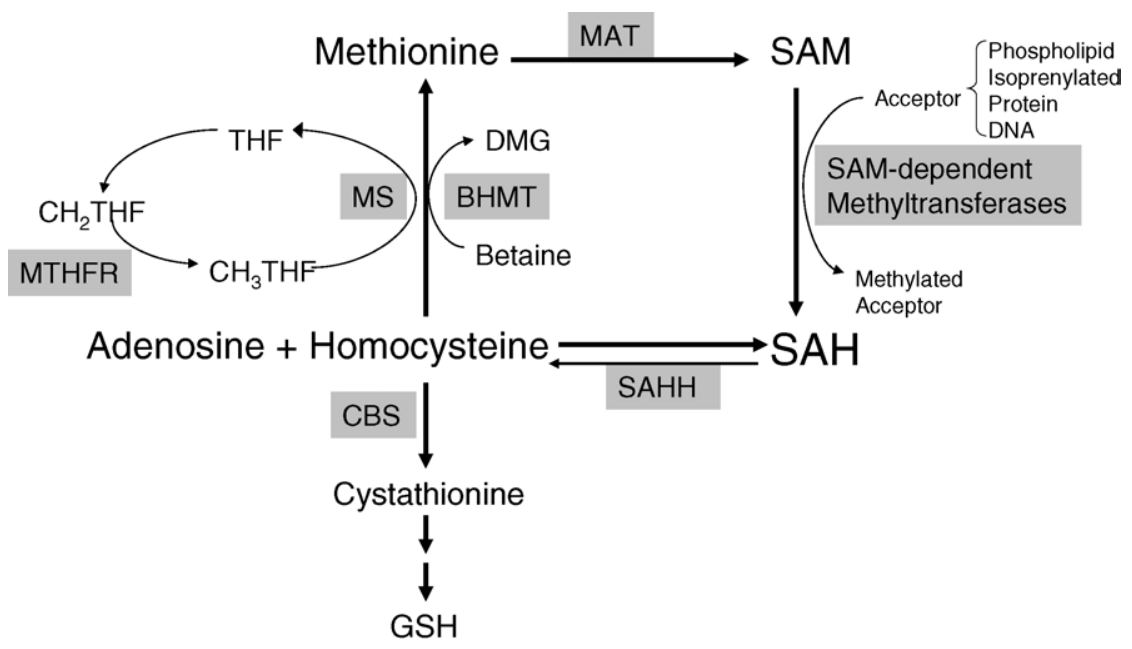

Fig. 1. Methionine metabolic pathway in the liver. Using ATP as a substrate, methionine adenosyltransferase (MAT) converts methionine to S-adenosylmethionine (SAM), which then serves as a methyl-group donor substrate for methyltransferases. The other product of methyltransferase reaction Sadenosylhomocysteine (SAH) is hydrolyzed to adenosine and homocysteine by S-adenosylhomocysteine hydrolase (SAHH). The thermodynamic of this reaction favors the formation of SAH if the products are not removed. The methionine cycle is completed when homocysteine is remethylated back to methionine by $\mathrm{B}_{12}$-dependent methionine synthase (MS) using 5-methyltetrahydrofolate $\left(\mathrm{CH}_{3} \mathrm{THF}\right)$ as substrate. The latter is generated by methylene tetrahydrofolate reductase (MTHFR). Homocysteine can also be remethylated to methionine by betaine homocysteine methyltransferase (BHMT) with the formation of dimethylglycine (DMG). Homocysteine can also be catabolized through the transulfuration pathway initiated by $\mathrm{B}_{6}$-dependent cystathionine $\beta$ synthase (CBS) to generate glutathione (GSH).

ing ethanol exposure drives SAH hydrolase to catalyze the energetically favorable reverse reaction resulting in increased SAH generation [22].

In recent years there have been reports of increased intracellular SAH levels inducing apoptosis in many different cell types [23-29]. Therefore, we put forth the hypothesis that increased intracellular SAH generation may be responsible for hepatocyte apoptosis seen after ethanol administration. To test our hypothesis, we needed to achieve elevated intracellular SAH levels in hepatocytes without ethanol exposure. We accomplished this undertaking by exploiting the unique characteristic of the enzyme SAH hydrolase - the only enzyme involved in SAH catabolism. Since SAH hydrolase catalyzes the reversible hydrolysis to adenosine and homocysteine with equilibrium of the reaction favoring the synthesis of SAH [30,31], altering the levels of its products (adenosine and/or homocysteine) has been shown to play a critical role in the control of tissue levels of SAH [24-26,32]. Therefore in this study, hepatocytes were exposed to varying concentrations of adenosine in order to promote the generation of $\mathrm{SAH}$ in order to increase intracellular SAH levels. Following exposure to adenosine, indices of apoptosis were determined.

In addition, our laboratory has also been interested in the therapeutic properties of betaine, a naturally occurring trimethylglycine that principally functions as an osmolyte and methyl donor [33]. As an osmolyte, betaine protects cells, proteins and enzymes from environmental stress, including extreme temperature, high salinity and low water content. As a methyl donor, betaine participates in the methionine cycle, and by remethylating homocysteine via betaine-homocysteine-methyltransferase (BHMT) has been shown to alleviate ethanol-induced changes in intracellular SAH levels [22].

Therefore, the objective of this study was: (1) to induce elevated intracellular SAH levels in hepatocytes without ethanol intervention and observe its effect on hepatocyte apoptosis and (2) to evaluate the therapeutic affect of betaine on hepatocyte apoptosis under these conditions.

\section{Materials and methods}

\subsection{Animals}

Male Wistar rats (Charles River Laboratories, Wilmington, MA) weighing 150-175 g were used. The animals were fed the Purina 5001 diet (Ralston Purina, St. Louis, MO) and housed at the Omaha Veterans Affairs Medical Center Animal Research Facility. The care and the use of the rats was approved by the Institutional Animal Care and Use Committee in accordance with the guidelines of the Institutional Care and Use Committee of the National Institute on Drug Abuse, National Institutes of Health, and the Guide for the Care and Use of Laboratory Animals [34].

\subsection{Hepatocyte isolation and experimental design}

Hepatocytes were obtained from the livers of the rats by a modified collagenase-perfusion technique as previously described [22]. Viabilities of the different cell populations were determined by trypan blue exclusion, and only cell preparations attaining a viability of $>90 \%$ were used. Isolated hepatocytes were plated (4.5 million viable 
cells/dish) on $10 \mu \mathrm{g}$ collagen IV-coated $100 \mathrm{~mm}$ culture dishes in Williams' medium $\mathrm{E}$ supplemented with antibiotics and $5 \%$ FCS. After $2 \mathrm{~h}$ of plating at $37{ }^{\circ} \mathrm{C}$ in a humidified atmosphere of $95 \% \mathrm{O}_{2} / 5 \% \mathrm{CO}_{2}$, the medium and unattached cells were removed and replaced with $10 \mathrm{ml}$ of serum-supplemented Williams' medium E containing $0-1 \mathrm{mmol} / \mathrm{L}$ adenosine. In some dishes, $2 \mathrm{mmol} / \mathrm{L}$ betaine and/or $10 \mathrm{mmol} / \mathrm{L}$ dimethylglycine (DMG) were added just before addition of adenosine. DMG is the demethylated product of betaine formed by the BHMT catalyzed reaction and is shown to be a potent feedback inhibitor of porcine, rat and human BHMT [35]. Betaine was obtained from Danisco USA Incorporated, Ardsley, NY. All other chemicals were obtained from SigmaAldrich Corp., St. Louis, MO.

After overnight treatment, the following analyses were done.

Extent of apoptosis was evaluated by the measurements of the following markers of apoptosis:

Caspase-3 activation assay: Treated hepatocytes were lysed and then assayed for specific caspase activity following manufacturers' instructions using commercially available caspase-3 Ac-DEVD-AMC fluorogenic substrate (BD Biosciences, San Diego, CA). Caspase activity was evaluated by measuring the release of AMC (7-Amino-4-methylcoumarin) obtained by the cleavage of the defined synthetic peptide sequence by caspase-3 using a Perkin-Elmer Luminescence Spectrophotometer LS 50B. Free AMC obtained from Sigma-Aldrich, St. Louis, MO was used as the standard. The caspase- 3 activity was expressed as pmoles of AMC produced per mg hepatocyte lysate protein.

DNA-fragmentation analysis: The treated hepatocytes were harvested, fixed in ethanol and incubated with DNA staining solution (RNAase A/propidium iodide mixture) [36]. After incubation, the nuclear DNA content in hepatocytes was evaluated using a FACSCalibur (excitation, 488; emission $575 \mathrm{~nm}$; Becton Dickinson, San Jose, CA) flow cytometer. DNA fluorescence pulse processing was used to discriminate between single cells and aggregates of cells by evaluating the FL2-width versus FL2-area scatter plot. Light scatter gating was used to eliminate smaller debris from analysis. DNA content was displayed on a linear scale. Apoptosis was determined by DNA hypoploidy quantification by identifying the percentage of cells in the sub-G1 hypodiploid zone of the DNA fluorescent cytogram using Modfit analysis program obtained from Varity software house (VSH, Topsham, ME). Data was collected from 10,000 nuclei/assay.

Measurement of cell necrosis: Cytotoxicity measurements were done by determining the release of lactate dehydrogenase $(\mathrm{LDH})$ in the culture medium. The leakage of LDH was measured by incubating a volume of the medium with $\mathrm{NADH}(0.2 \mathrm{mmol} / \mathrm{L})$ and pyruvic acid $(0.4 \mathrm{mmol} / \mathrm{L})$ diluted in phosphate buffer as described [37] and expressed as \% LDH release over controls.
SAH levels: Hepatocyte pellets were homogenized in $0.5 \mathrm{~mol} / \mathrm{L} \mathrm{HClO}_{4}$, and the filtered acid extracts directly subjected to HPLC analysis for the determination of hepatocellular SAH levels following the procedure of $\mathrm{Fu}$ et al. [38] as detailed in our publication [22].

Homocysteine levels: Hepatocyte pellets and the conditioned media were subjected to HPLC analysis for the determination of total (hepatocellular + secreted) homocysteine [39] as detailed in our publication [22].

Statistical analysis: Data were analyzed by ANOVA, followed by Student's Newman-Keuls post hoc test. A $P$ value $<0.05$ was regarded as statistically significant.

\section{Results}

Hepatocytes cultured in vitro overnight on collagen IV matrix in serum-supplemented Williams' medium E maintained their normal phenotype with flattened morphology. In dishes with adenosine supplementation, the hepatocytes showed changes in morphology to a more rounded appearance.

The caspases are a family of cysteine-aspartic acidspecific proteases involved in the cleavage of cellular substrates that ultimately leads to apoptosis. This family contains some members that act as signal transduction molecules, while other members carry out the executioner function in the process of apoptosis. The measurement of executioner caspase activation (caspase-3) has become a widely accepted marker of the process of apoptosis. We observed that incubation with increasing concentrations of adenosine induced a dose-dependent increase in caspase-3 activity in hepatocytes (Fig. 2). An 8-10-fold increase in its activity was seen with the highest dose of adenosine tested

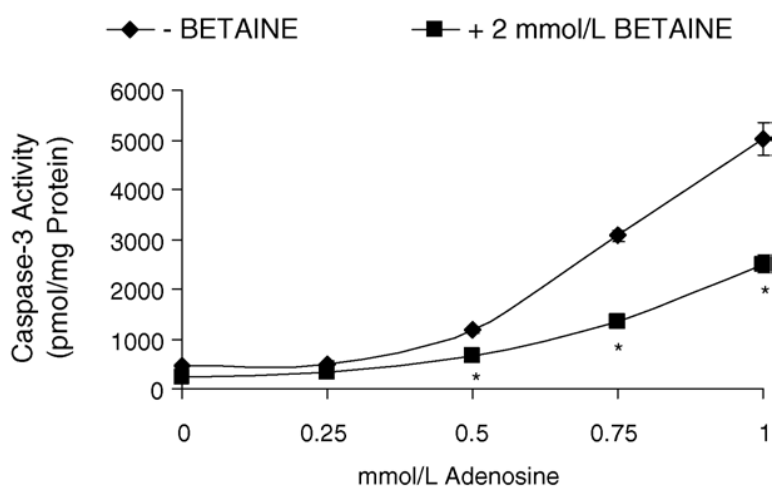

Fig. 2. Effect of adenosine and betaine on caspase-3 activity in rat hepatocytes. Isolated hepatocytes from chow-fed rats were seeded on collagen IV-coated $100 \mathrm{~mm}$ petri plates at 4.5 million cells/plate in Williams' medium E supplemented with 5\% FCS. After $2 \mathrm{~h}$ of plating, the unattached cells were removed and replaced with serum-supplemented Williams' medium E containing varying amounts of adenosine (0$1 \mathrm{mmol} / \mathrm{L}$ ) in the presence and absence of $2 \mathrm{mmol} / \mathrm{L}$ betaine. After overnight incubation, caspase-3 activity was determined in the cellular lysate. Data represent mean of five determinations. $* P<0.05$ as compared to betaine-untreated samples. 
( $1 \mathrm{mmol} / \mathrm{L}$ ). When betaine at $2 \mathrm{mmol} / \mathrm{L}$ was added along with adenosine, a 40-50\% decrease in adenosine-induced caspase- 3 activity increase was seen.

Apoptosis was also quantified by determining the extent of internucleosomal DNA cleavage or DNA-fragmentation by subjecting the treated hepatocytes to flow cytometric analysis following PI staining. By this methodology, the apoptotic nuclei stained with PI appeared as a broad hypodiploid peak that was easily distinguishable from viable nuclei with diploid DNA. DNA-fragmentation or percent apoptosis determined from the cytograms obtained from one representative experiment is shown in Fig. 3. The average of the percent apoptosis obtained from five such independent experiments are shown as Table 1. We observed low percent apoptosis to the extent of $2.6 \pm 0.5 \%$ in freshly isolated hepatocytes. There was a slight enhancement of percent apoptosis in hepatocytes cultured overnight under basal conditions. Treatment with $0.75 \mathrm{mmol} / \mathrm{L}$ adenosine led to a $\sim 3$-fold increase in apoptosis as compared to controls. Co-incubation with betaine led to a significant decrease in DNA-fragmentation in adenosine-exposed hepatocytes to near control levels (Fig. 3). These flow cytometric results correlated with the results obtained for caspase activities $\left(r^{2}=0.91\right.$, $P<0.05)$.

Since one pathway of intracellular adenosine metabolism relates to increased intracellular SAH levels, these levels were determined in hepatocyte pellets after treatments. We observed that when rat hepatocytes were incu-
Table 1

Effects of adenosine and betaine on hepatocyte apoptosis

\begin{tabular}{ll}
\hline Treatment & Percent apoptosis $^{\mathrm{a}}$ \\
\hline Before incubation & $2.6 \pm 1.9 \mathrm{a}$ \\
After overnight incubation with & $7.4 \pm 2.2 \mathrm{a}$ \\
$\quad$ Media alone & $4.2 \pm 1.2 \mathrm{a}$ \\
$\quad$ +Betaine $(2 \mathrm{mmol} / \mathrm{L})$ & $19.2 \pm 4.2 \mathrm{~b}$ \\
$\quad$ +Adenosine $(0.75 \mathrm{mmol} / \mathrm{L})$ & $8.6 \pm 3.1 \mathrm{a}$ \\
$\quad+$ Adenosine $(0.75 \mathrm{mmol} / \mathrm{L})+$ & \\
betaine $(2 \mathrm{mmol} / \mathrm{L})$ & \\
\hline
\end{tabular}

Rat hepatocytes, either freshly isolated or following overnight culture in the presence and absence of $0.75 \mathrm{mmol} / \mathrm{L}$ adenosine and $2 \mathrm{mmol} / \mathrm{L}$ betaine, were fixed in ethanol, incubated with DNA staining solution and the nuclear DNA content in hepatocytes evaluated using a FACSCalibur (excitation, 488; emission $575 \mathrm{~nm}$ ) flow cytometer. Apoptosis was determined by DNA hypoploidy quantification by identifying the percentage of cells in the subG1 hypodiploid zone of the DNA fluorescent cytogram using Modfit analysis program obtained from Varity software house (VSH, Topsham, ME).

${ }^{a}$ Values listed are means \pm S.E.M. for five independent experiments. Values not sharing a common letters ( $a$ and $b$ ) are significantly different by ANOVA; $P<0.01$.

bated with varying amounts of adenosine, a corresponding increase in intracellular SAH levels was seen (Fig. 4). A 10 -fold increased level was observed with $1 \mathrm{mmol} / \mathrm{L}$ adenosine treatment. While adenosine treatment increased SAH levels, the presence of adenosine decreased the total homocysteine levels. The homocysteine values (mean \pm S.E.M.) for controls were $675 \pm 70 \mathrm{pmol} / \mu \mathrm{g}$ DNA compared to values for $0.25,0.5,0.75$ and $1 \mathrm{mmol} / \mathrm{L}$ adenosine
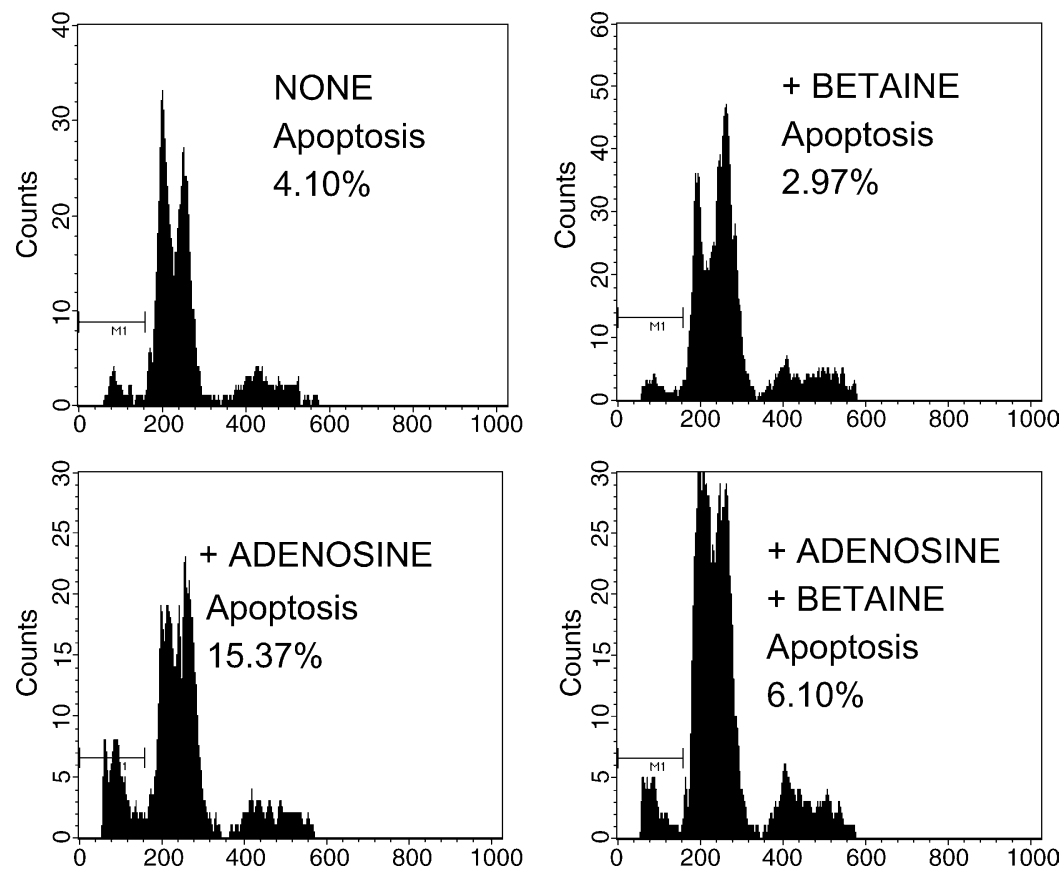

Fig. 3. Flow cytograms of DNA-fragmentation in hepatocytes: effects of adenosine and betaine. Rat hepatocytes seeded on collagen IV-coated $100 \mathrm{~mm}$ petri plates in Williams' medium E supplemented with 5\% FCS were treated overnight in the presence and absence of $0.75 \mathrm{mmol} / \mathrm{L}$ adenosine and $2 \mathrm{mmol} / \mathrm{L}$ betaine. Following treatment, the hepatocytes were harvested, fixed in ethanol, incubated with DNA staining solution and the nuclear DNA content evaluated using a FACSCalibur flow cytometer. Apoptosis was determined by DNA hypoploidy quantification by identifying the percentage of cells in the sub-G1 hypodiploid zone (recognized at the left of the major peak, labeled M1 in the cytogram) using Modfit analysis program obtained from Varity software house (VSH, Topsham, ME). The cytograms and the percent apoptosis are from one of five independent experiments, all of which had similar results. 


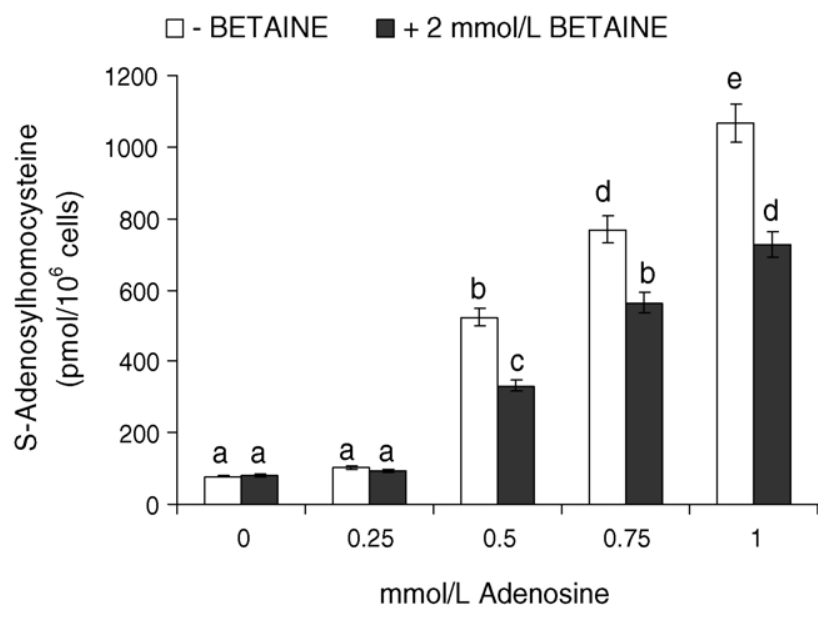

Fig. 4. Effect of adenosine and betaine on intracellular S-adenosylhomocysteine (SAH) levels. Isolated hepatocytes from chow-fed rats were seeded on collagen IV-coated $100 \mathrm{~mm}$ petri plates at 4.5 million cells/plate in Williams' medium E supplemented with 5\% FCS. After $2 \mathrm{~h}$ of plating, the unattached cells were removed and replaced with serum-supplemented Williams' medium E containing varying amounts of adenosine (0$1 \mathrm{mmol} / \mathrm{L}$ ) in the presence and absence of $2 \mathrm{mmol} / \mathrm{L}$ betaine. Following overnight incubations, the intracellular SAH levels were determined. Data represent mean of three determinations. Values not sharing a common subscript letter are significantly different by ANOVA; $P<0.05$.

of $430 \pm 122,351 \pm 174,303 \pm 177$ and $292 \pm 112 \mathrm{pmol} /$ $\mu \mathrm{g}$ DNA, respectively $(P<0.05)$.

We further observed that adenosine-induced increases in SAH levels were significantly decreased by $40-50 \%$ following co-incubation with betaine, indicating that betaine may be protecting against adenosine-induced apoptosis presumably via BHMT catalyzed methylation reactions to decrease SAH levels.

Therefore, to evaluate whether the protective mechanism of betaine incubation is via methylation reactions, hepatocytes were simultaneously also exposed to DMG, a potent inhibitor of BHMT activity. Addition of DMG alone to control hepatocytes or adenosine-treated hepatocytes resulted in a small increase in caspase-3 activity (Fig. 5). More importantly, it attenuated the protective effect of betaine under basal as well as under adenosine-supplemented condition.

No significant changes in LDH release under any experimental conditions were observed (data not shown).

\section{Discussion}

This study was conducted with the goal to causally link elevated intracellular SAH levels seen in hepatocytes following ethanol consumption to alcoholic apoptosis observed in a variety of animal species. In this study, we conducted our experiments on isolated hepatocytes obtained from chow-fed rats rather than ethanol-fed rats because we wanted hepatocytes without any previous alterations in pathways of methionine metabolism. These naïve hepatocytes were then exposed in vitro to agents that
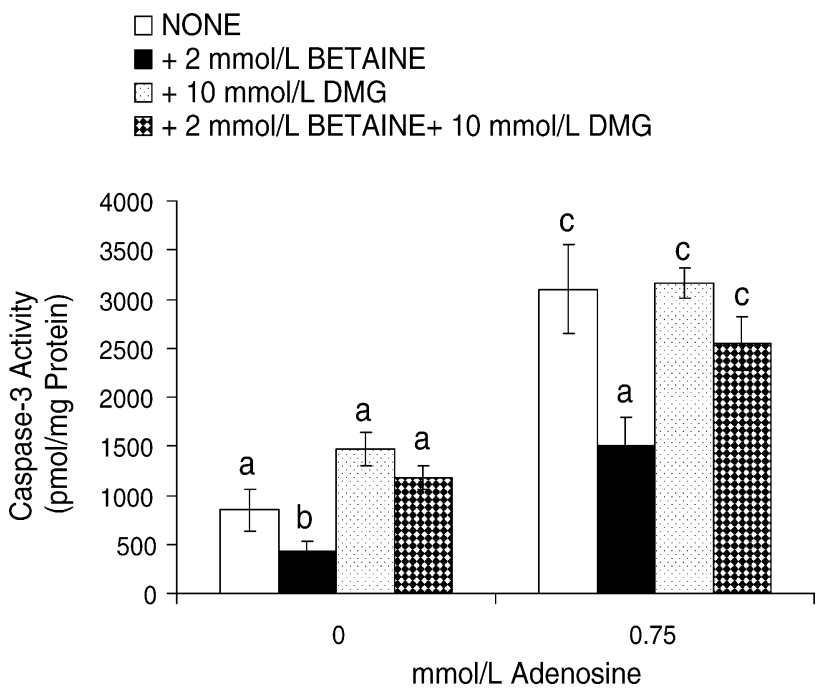

Fig. 5. Effect of adenosine, betaine and dimethylglycine (DMG) on caspase-3 activity. Isolated hepatocytes from chow-fed rats were seeded on collagen IV-coated $100 \mathrm{~mm}$ petri plates at 4.5 million cells/plate in Williams' medium E supplemented with $5 \%$ FCS. After $2 \mathrm{~h}$ of plating, the unattached cells were removed and replaced with serum-supplemented Williams' medium $\mathrm{E}$ in the presence and absence of $0.75 \mathrm{mmol} / \mathrm{L}$ adenosine, $2 \mathrm{mmol} / \mathrm{L}$ betaine and $10 \mathrm{mmol} / \mathrm{L}$ DMG. After overnight incubation, caspase-3 activity was determined in the cellular lysate. Data represent mean of five determinations. Values not sharing a common subscript letter are significantly different by ANOVA; $P<0.05$.

are known to increase intracellular SAH levels by exploiting the unique characteristic of the enzyme, SAH hydrolase, which catalyzes the reverse hydrolysis of SAH to adenosine and homocysteine. Incubations with SAHhydrolase inhibitor, or under conditions of excess adenosine and homocysteine either added alone or in combination have all been shown to generate increased intracellular SAH [24-26,32]. But in our study, we did not use homocysteine as an agent to achieve increased intracellular SAH levels but instead focused on adenosine. This agent elevated intracellular SAH without increasing either hepatocellular or secreted homocysteine levels, thus facilitating the effects of elevated intracellular SAH to be distinguished from those implicated via elevated homocysteine-induced ER stress $[12,13]$.

The data in this study indicate that increased intracellular SAH functions as a physiological modulator of hepatocyte apoptosis. Our study also corroborates a recent publication that implicated increased intracellular SAH levels with hepatotoxicity [40] and supports of the concept that removal of intracellular SAH is a potential therapeutic option. Although the message is similar, this recent published study differs from ours in that no toxicity was observed in HepG2 on exposure to agents, such as adenosine, homocysteine and 3-deaza-adenosine (a SAH hydrolase inhibitor) that are known to increase intracellular SAH levels [40]. These HepG2 cells with elevated SAH levels, however, showed increased sensitivity to TNF $\alpha$ toxicity [40]. The disparity between their study and our study may be due to the fact that Song et al. conducted their 
experiments in a hepatoma-derived cell line and not with hepatocytes in primary culture that were employed in the current study. Recent reports from our laboratory have also shown that exposure to 7-deaza-adenosine (another potent SAH hydrolase inhibitor) alone also induces apoptosis in rat hepatocytes in primary cultures [41].

We further show that betaine confers protection against apoptosis. This evidence is based on the following observations. First, addition of adenosine that increase intracellular SAH levels, induce increases in activity of effector caspase (caspase-3) as well as cause increases in DNAfragmentation. A linear correlation existed between $\mathrm{SAH}$ levels and caspase- 3 activity $\left(r^{2}=0.91 ; P<0.01\right)$. Second, betaine by decreasing intracellular $\mathrm{SAH}$ by virtue of remethylating homocysteine, results in significant attenuation in adenosine-induced increased caspase activities and DNA-fragmentation.

In our hands, the minimum concentration of adenosine required to observe increases in intracellular SAH levels in cultured hepatocytes was $0.5 \mathrm{mmol} / \mathrm{L}$, which was also the minimum concentration required to observe increases in caspases activation. Earlier studies had employed much lower amounts of adenosine $(\sim 50 \mu \mathrm{mol} / \mathrm{L})$ in order to achieve elevated intracellular SAH and induce toxicity in cultured mouse lymphoblasts [23]. It is possible that the use of different cell-types or animal sources may be responsible for the inconsistency observed between the two studies. This speculation is based on the fate of adenosine metabolism that relates not only to homocysteine metabolism and the ability of adenosine to form a stable complex with SAH hydrolase to inhibit SAH hydrolase activity, but also to the metabolism of adenosine within seconds by adenosine deaminase (ADA) to inosine or its phosphorylation by adenosine kinase to form $5^{\prime}$ AMP. It is likely that lower adenosine concentration could induce toxicity in lymphoblasts in previous studies because these cells were isolated from ADA-deficient mice and therefore were probably more susceptible to lower adenosine concentrations because of its inhibited metabolism to inosine.

More recent studies using various cell types that implicate increased adenosine toxicity via increased SAH levels have employed comparable adenosine concentrations as used by us [24,26-29,42]. The mechanism of action of adenosine appears to be via its ability to act as a substrate for as well as an inhibitor of SAH hydrolase thereby elevating intracellular SAH.

Further implicating the role of elevated SAH as one of the principle factors responsible for apoptosis were the experiments conducted with betaine supplementation. Betaine by virtue of decreasing elevated SAH levels as shown in this study as well as in our previous publication [22], also significantly attenuated-induced apoptosis. Additional experiments conducted with the inclusion of excess DMG-a potent feedback inhibitor of porcine, rat and human BHMT [35] - demonstrated the mechanism of action of betaine. We observed that DMG added in 5-fold excess of the concentration of betaine partially blocked the protective effect of betaine on adenosine-induced apoptosis. This reaffirmed that the protective effect of betaine was primarily via methyl-group transfer reaction catalyzed by BHMT that alleviates the increased intracellular SAH levels by remethylating homocysteine.

The protective effect of betaine in preventing hepatocyte apoptosis has also been corroborated by reports from different laboratories in which apoptosis was induced by various conditions including hypertonicity [43], bile salts [44] and perfusion-reperfusion liver injury [45]. It is not known whether these experimental manipulations of hypertonicity, bile salts exposure and perfusion-reperfusion injury also result in alterations in methionine metabolic pathways, especially elevations of intracellular SAH levels.

Betaine has also been shown to protect against alcoholic liver injury $[12,13,20,22,46-49]$, prevent carbon tetrachloride-induced liver injury [50] as well as to considerably decrease indices of steatosis in NASH patients [51,52]. In addition, betaine has been effectively used as a treatment regimen for patients with inherited genetic disorders of hyperhomocysteinemia such as cystathionine $\beta$-synthase deficiency and methylene tetrahydrofolate reductase deficiency [53]. Although the untreated patients with these deficiencies always present with hepatic abnormalities, of particular interest to us is the generation of animal models with these genetic deficiencies where a more detailed biochemical analysis has been done. The livers of these animals have high SAH levels [54-56], show liver injury [57] and betaine administration appears to reverse these biochemical alterations [57].

Our future studies are aimed at elucidating the mechanism by which elevated intracellular SAH promotes apoptosis. Since the homeostatic levels of SAH are critically important on account of SAH being a potent productinhibitor of many SAM-dependent methyltransferases [58], we postulate a critical role of one or more liver methyltransferases in preventing hepatocyte apoptosis. Recent studies have shown that the carboxyl methylation reaction of small GTPases is a crucial activation step that facilitates these proteins to participate in anti-apoptotic signaling pathways [59]. We speculate that inhibition of the critical methyltransferase (such as isoprenyl cysteine methyltransferase) by elevated intracellular SAH levels may be responsible for adenosine-induced apoptosis in hepatocytes.

To conclude, we have data that provides a definite link between elevated intracellular SAH and increased hepatocyte apoptosis and presents a therapeutic approach of betaine as a modality to prevent alcoholic apoptosis. These results indicate that the elevation of SAH levels in the liver by ethanol appears to be a major factor in altering methylation reactions and in increasing apoptosis in the liver. Considering the link between hepatocyte apoptosis and liver fibrosis [60], inhibition of hepatocyte apoptosis by betaine may also be an anti-fibrotic therapeutic strategy. 


\section{References}

[1] Benedetti A, Brunelli E, Risicato R, Cilluffo T, Jezequel AM, Orlandi F. Subcellular changes and apoptosis induced by ethanol in rat liver. J Hepatol 1988;6:137-43.

[2] Baroni GS, Marucci L, Benedetti A, Mancini R, Jezequel AM, Orlandi F. Chronic ethanol feeding increases apoptosis and cell proliferation in rat liver. J Hepatol 1994;20:508-13.

[3] Halsted CH, Villanueva J, Chandler CJ, Stabler SP, Allen RH, Muskhelishvili L, et al. Ethanol feeding of micropigs alters methionine metabolism and increases hepatocellular apoptosis and proliferation. Hepatology 1996;23:497-505.

[4] Higuchi H, Kurose I, Kato S, Miura S, Ishii H. Ethanol-induced apoptosis and oxidative stress in hepatocytes. Alcohol Clin Exp Res 1996;20:340A-6A.

[5] Nanji AA. Apoptosis and alcoholic liver disease. Semin Liver Dis 1998;18:187-90.

[6] Casey CA, Nanji A, Cederbaum AI, Adachi M, Takahashi T. Alcoholic liver disease and apoptosis. Alcohol Clin Exp Res 2001;25:49S-53S.

[7] Deaciuc IV, Fortunato F, D'Souza NB, Hill DB, McClain CJ. Chronic alcohol exposure of rats exacerbates apoptosis in hepatocytes and sinusoidal endothelial cells. Hepatol Res 2001;19:306-24.

[8] Deaciuc II, D’Souza NB, Fortunato F, Hill DB, Sarphie TG, McClain CJ. Alcohol-induced sinusoidal endothelial cell dysfunction in the mouse is associated with exacerbated liver apoptosis and can be reversed by caspase inhibition. Hepatol Res 2001;19:85-97.

[9] Deaciuc IV, D'Souza NB, de Villiers WJ, Burikhanov R, Sarphie TG, Hill DB, et al. Inhibition of caspases in vivo protects the rat liver against alcohol-induced sensitization to bacterial lipopolysaccharide. Alcohol Clin Exp Res 2001;25:935-43.

[10] Cardin R, D'Errico A, Fiorentino M, Cecchetto A, Naccarato R, Farinati F. Hepatocyte proliferation and apoptosis in relation to oxidative damage in alcohol-related liver disease. Alcohol Alcohol 2002;37:43-8.

[11] Deaciuc IV, D'Souza NB, Burikhanov R, Nasser MS, Voskresensky IV, De Villiers WJ, et al. Alcohol, but not lipopolysaccharide-induced liver apoptosis involves changes in intracellular compartmentalization of apoptotic regulators. Alcohol Clin Exp Res 2004;28:160-72.

[12] Ji C, Kaplowitz N. Betaine decreases hyperhomocysteinemia, endoplasmic reticulum stress, and liver injury in alcohol-fed mice. Gastroenterology 2003;124:1488-99.

[13] Ji C, Deng Q, Kaplowitz N. Role of TNF-alpha in ethanol-induced hyperhomocysteinemia and murine alcoholic liver injury. Hepatology 2004;40:442.

[14] Barak AJ, Beckenhauer HC, Tuma DJ, Badakhsh S. Effects of prolonged ethanol feeding on methionine metabolism in rat liver. Biochem Cell Biol 1987;65:230-3.

[15] Finkelstein JD. Methionine metabolism in mammals. J Nutr Biochem 1990;1:228-37.

[16] Tsukamoto H, Lu SC. Current concepts in the pathogenesis of alcoholic liver injury. FASEB J 2001;15:1335-49.

[17] Barak AJ, Beckenhauer HC, Tuma DJ. Ethanol feeding inhibits the activity of N5-methyltetrahydrofolate-homocysteine methyltransferase in the rat. IRCS Med Sci 1985;13:760-1.

[18] Barak AJ, Beckenhauer HC, Tuma DJ. Methionine synthase: a possible prime site of the ethanolic lesion in liver. Alcohol 2002;26:65-7.

[19] Lieber CS, Casini A, DeCarli LM, Kim CI, Lowe N, Sasaki R, et al. Sadenosyl-L-methionine attenuates alcohol-induced liver injury in the baboon. Hepatology 1990;11:165-72.

[20] Barak AJ, Beckenhauer HC, Kharbanda KK, Tuma DJ. Chronic ethanol consumption increases homocysteine accumulation in hepatocytes. Alcohol 2001;25:77-81.

[21] Halsted CH, Villanueva JA, Devlin AM, Niemela O, Parkkila S, Garrow TA, et al. Folate deficiency disturbs hepatic methionine metabolism and promotes liver injury in the ethanol-fed micropig. Proc Natl Acad Sci USA 2002;99:10072-7.
[22] Barak AJ, Beckenhauer HC, Mailliard ME, Kharbanda KK, Tuma DJ. Betaine lowers elevated S-adenosylhomocysteine levels in hepatocytes from ethanol-fed rats. J Nutr 2003;133:2845-8.

[23] Kredich NM, Hershfield MS. S-adenosylhomocysteine toxicity in normal and adenosine kinase-deficient lymphoblasts of human origin. Proc Natl Acad Sci USA 1979;76:2450-4.

[24] Skinner MA, Ho HJ, Chan VL. Inhibition of methylation of DNA and tRNA by adenosine in an adenosine-sensitive mutant of the baby hamster kidney cell line. Arch Biochem Biophys 1986;246:725-32.

[25] Ratter F, Germer M, Fischbach T, Schulze-Osthoff K, Peter ME, Droge $\mathrm{W}$, et al. S-adenosylhomocysteine as a physiological modulator of Apo-1-mediated apoptosis. Int Immunol 1996;8:1139-47.

[26] Rounds S, Yee WL, Dawicki DD, Harrington E, Parks N, Cutaia MV. Mechanism of extracellular ATP- and adenosine-induced apoptosis of cultured pulmonary artery endothelial cells. Am J Physiol 1998; 275: L379-L388.

[27] Zhao Z, Kapoian T, Shepard M, Lianos EA. Adenosine-induced apoptosis in glomerular mesangial cells. Kidney Int 2002;61:1276-85.

[28] Bellas RE, Harrington EO, Sheahan KL, Newton J, Marcus C, Rounds S. FAK blunts adenosine-homocysteine-induced endothelial cell apoptosis: requirement for PI 3-kinase. Am J Physiol Lung Cell Mol Physiol 2002;282:L1135-42.

[29] Harrington EO, Smeglin A, Parks N, Newton J, Rounds S. Adenosine induces endothelial apoptosis by activating protein tyrosine phosphatase: a possible role of p38alpha. Am J Physiol Lung Cell Mol Physiol 2000;279:L733-42.

[30] Kloor D, Ludtke A, Stoeva S, Osswald H. Adenosine binding sites at S-adenosylhomocysteine hydrolase are controlled by the NAD+/ NADH ratio of the enzyme. Biochem Pharmacol 2003;66:2117-23.

[31] Prigge ST, Chiang PK. S-adenosylhomocysteine hydrolase. In: Carmel R, Jacobsen DW, editors. Homocysteine in Health and Disease. Cambridge: Cambridge University Press; 2001. p. 79-91.

[32] Bergmann S, Shatrov V, Ratter F, Schiemann S, Schulze-Osthoff K, Lehmann V. Adenosine and homocysteine together enhance TNFmediated cytotoxicity but do not alter activation of nuclear factorkappa B in L929 cells. J Immunol 1994;153:1736-43.

[33] Craig SA. Betaine in human nutrition. Am J Clin Nutr 2004;80:53949.

[34] Guide for the Care and Use of Laboratory Animals. National Academy Press, 1996.

[35] Garrow TA. Purification, kinetic properties, and cDNA cloning of mammalian betaine-homocysteine methyltransferase. J Biol Chem 1996;271:22831-8.

[36] Darzynkiewicz Z, Traganos F. Measurement of apoptosis. Adv Biochem Eng Biotechnol 1998;62:33-73.

[37] Bergmeyer HU, Bernt E. Lactate Dehydrogenase. UV assay with pyruvate and NADH. Meth Enzymatic Anal 1974;2:574-8.

[38] Fu W, Dudman NP, Perry MA, Young K, Wang XL. Interrelations between plasma homocysteine and intracellular S-adenosylhomocysteine. Biochem Biophys Res Commun 2000;271:47-53.

[39] Cornwell PE, Morgan SL, Vaughn WH. Modification of a highperformance liquid chromatographic method for assay of homocysteine in human plasma. J Chromatogr 1993;617:136-9.

[40] Song Z, Zhou Z, Uriarte S, Wang L, Kang YJ, Chen T, et al. Sadenosylhomocysteine sensitizes to TNF-alpha hepatotoxicity in mice and liver cells: a possible etiological factor in alcoholic liver disease. Hepatology 2004;40:989-97.

[41] Kharbanda KK, Rogers 2nd DD, Beckenhauer HC, Siford GL, Barak AJ, Mailliard ME, et al. Tubercidin-induced apoptosis via increased hepatocellular levels of S-adenosylhomocysteine is attenuated by betaine administration. Alcohol Clin Exp Res 2005;29:182A.

[42] Dawicki DD, Chatterjee D, Wyche J, Rounds S. Extracellular ATP and adenosine cause apoptosis of pulmonary artery endothelial cells. Am J Physiol 1997;273:L485-94.

[43] Horio M, Ito A, Matsuoka Y, Moriyama T, Orita Y, Takenaka M, et al. Apoptosis induced by hypertonicity in Madin Darley canine kidney 
cells: protective effect of betaine. Nephrol Dial Transplant 2001; 16:483-90.

[44] Graf D, Kurz AK, Reinehr R, Fischer R, Kircheis G, Haussinger D. Prevention of bile acid-induced apoptosis by betaine in rat liver. Hepatology 2002;36:829-39.

[45] Wettstein M, Haussinger D. Cytoprotection by the osmolytes betaine and taurine in ischemia-reoxygenation injury in the perfused rat liver. Hepatology 1997;26:1560-6.

[46] Barak AJ, Beckenhauer HC, Junnila M, Tuma DJ. Dietary betaine promotes generation of hepatic $\mathrm{S}$-adenosylmethionine and protects the liver from ethanol-induced fatty infiltration. Alcohol Clin Exp Res 1993;17:552-5.

[47] Barak AJ, Beckenhauer HC, Tuma DJ. S-adenosylmethionine generation and prevention of alcoholic fatty liver by betaine. Alcohol 1994;11:501-3.

[48] Barak AJ, Beckenhauer HC, Badakhsh S, Tuma DJ. The effect of betaine in reversing alcoholic steatosis. Alcohol Clin Exp Res 1997; 21:1100-2.

[49] Kanbak G, Inal M, Baycu C. Ethanol-induced hepatotoxicity and protective effect of betaine. Cell Biochem Funct 2001;19:281-5.

[50] Junnila M, Barak AJ, Beckenhauer HC, Rahko T. Betaine reduces hepatic lipidosis induced by carbon tetrachloride in Sprague-Dawley rats. Vet Hum Toxicol 1998;40:263-6.

[51] Abdelmalek MF, Angulo P, Jorgensen RA, Sylvestre PB, Lindor KD. Betaine, a promising new agent for patients with nonalcoholic steatohepatitis: results of a pilot study. Am J Gastroenterol 2001;96:27117.

[52] Miglio F, Rovati LC, Santoro A, Setnikar I. Efficacy and safety of oral betaine glucuronate in non-alcoholic steatohepatitis. A double-blind, randomized, parallel-group, placebo-controlled prospective clinical study. Arzneimittelforschung 2000;50:722-7.
[53] Zavad'akova P, Fowler B, Zeman J, Suormala T, Pristoupilova K, Kozich V. CblE type of homocystinuria due to methionine synthase reductase deficiency: clinical and molecular studies and prenatal diagnosis in two families. J Inherit Metab Dis 2002;25:461-76.

[54] Chen Z, Karaplis AC, Ackerman SL, Pogribny IP, Melnyk S, LussierCacan S, et al. Mice deficient in methylenetetrahydrofolate reductase exhibit hyperhomocysteinemia and decreased methylation capacity, with neuropathology and aortic lipid deposition. Hum Mol Genet 2001;10:433-43.

[55] Caudill MA, Wang JC, Melnyk S, Pogribny IP, Jernigan S, Collins $\mathrm{MD}$, et al. Intracellular S-adenosylhomocysteine concentrations predict global DNA hypomethylation in tissues of methyl-deficient cystathionine beta-synthase heterozygous mice. J Nutr 2001; 131:2811-8.

[56] Choumenkovitch SF, Selhub J, Bagley PJ, Maeda N, Nadeau MR, Smith DE, et al. In the cystathionine beta-synthase knockout mouse, elevations in total plasma homocysteine increase tissue S-adenosylhomocysteine, but responses of S-adenosylmethionine and DNA methylation are tissue specific. J Nutr 2002;132:2157-60.

[57] Schwahn BC, Chen Z, Laryea MD, Wendel U, Lussier-Cacan S, Genest $\mathrm{Jr} \mathrm{J}$, et al. Homocysteine-betaine interactions in a murine model of 5, 10-methylenetetrahydrofolate reductase deficiency. FASEB J 2003;17:512-4.

[58] Clarke S, Banfield K. S-adenosylmethionine-dependent methyl transferases. In: Carmel R, Jacobsen DW, editors. Homocysteine in health and disease. Cambridge: Cambridge University Press; 2001 p. 63-78.

[59] Kramer K, Harrington EO, Lu Q, Bellas R, Newton J, Sheahan KL, et al. Isoprenylcysteine carboxyl methyltransferase activity modulates endothelial cell apoptosis. Mol Biol Cell 2003;14:848-57.

[60] Higuchi H, Gores GJ. Mechanisms of liver injury: an overview. Curr Mol Med 2003;3:483-90. 